112-pixel arrays of

high-efficiency STJ X-ray

detectors

S. Friedrich, J. Harris, W. K. Warburton, M. H.

Carpenter, J. A. Hall, R. Cantor

July 17,2013

15th International Workshop on Low Temperature Detectors (LTD-15)

Pasadena, CA, United States

June 24, 2013 through June 28, 2013 
This document was prepared as an account of work sponsored by an agency of the United States government. Neither the United States government nor Lawrence Livermore National Security, LLC, nor any of their employees makes any warranty, expressed or implied, or assumes any legal liability or responsibility for the accuracy, completeness, or usefulness of any information, apparatus, product, or process disclosed, or represents that its use would not infringe privately owned rights. Reference herein to any specific commercial product, process, or service by trade name, trademark, manufacturer, or otherwise does not necessarily constitute or imply its endorsement, recommendation, or favoring by the United States government or Lawrence Livermore National Security, LLC. The views and opinions of authors expressed herein do not necessarily state or reflect those of the United States government or Lawrence Livermore National Security, LLC, and shall not be used for advertising or product endorsement purposes. 


\title{
112-pixel arrays of high-efficiency STJ X-ray detectors
}

\author{
S. Friedrich ${ }^{1}$, J. Harris ${ }^{2}$, W. K. Warburton ${ }^{2}$, \\ M. H. Carpenter ${ }^{1,3}$, J. A. Hall ${ }^{3}$, and R. Cantor ${ }^{3}$ \\ ${ }^{1}$ Lawrence Livermore National Laboratory, Livermore, CA 94550, USA \\ ${ }^{2} X I A$ LLC, Hayward, CA 94544, USA \\ ${ }^{3}$ STAR Cryoelectronics, Santa Fe, NM 87508, USA
}

We are developing the next generation of high-resolution high-speed X-ray detectors based on superconducting tunnel junctions (STJS). They consist of 112-pixel arrays of $208 \mu \mathrm{m} \times 208 \mu \mathrm{m}$ Ta-Al-AlOx-Al-Ta tunnel junctions whose Ta absorber increases the detection efficiency compared to earlier Nb-based STJs. To read out these medium size detector arrays we have also developed a compact and scalable 32-channel preamplifier with an input voltage noise $<1 \mathrm{nV} / \sqrt{ } \mathrm{Hz}$ and a dc voltage bias for stable STJ operation between Fiske mode resonances. The pixels have a uniform response across the array, an energy resolution between 7.5 and $9.5 \mathrm{eV} \mathrm{FWHM} \mathrm{at} 525 \mathrm{eV}$, and can be operated at several 1000 counts/s per pixel.

\section{INTRODUCTION}

$\mathrm{X}$-ray absorption spectroscopy (XAS) is widely used at synchrotron light sources to characterize the electronic structure of materials. For XAS, the energy of the monochromatic X-ray beam is scanned across an electron binding energy of the element of interest, and the absorption is measured in transmission, by total electron yield (TEY) or by partial fluorescence yield (PFY). PFY XAS enables the analysis of dilute sample by discriminating against most of the X-ray background and recording just the weak fluorescence line of interest as a measure of absorption. This requires an Xray detector with sufficient solid angle coverage to capture as much of the weak fluorescence signal as possible, with sufficient energy resolution to separate this fluorescence line from the X-ray background due to other elements in the sample, and with sufficient speed to handle the total X-ray fluorescence at modern synchrotron beam lines.

Superconducting tunnel junction (STJ) X-ray detectors provide an advantage for XAS in the soft X-ray band whenever traditional Si or Ge detectors lack energy resolution and grating spectrometers lack solid angle coverage to separate a weak fluorescence signal from the X-ray background 


\section{S. Friedrich et al.}

efficiently [1, 2]. For this reason, Nb-based STJs have been used for a decade in bioenvironmental and material science applications at the synchrotron [1, 3-5]. More recently, STAR Cryoelectronics has developed STJs based on tantalum, since its high atomic number $\left(\mathrm{Z}_{\mathrm{Ta}}=73\right)$ increases the quantum efficiency, and its small energy gap $\left(\Delta_{\mathrm{Ta}}=0.7 \mathrm{meV}\right)$ improves the energy resolution over earlier Nb-based STJs. These Ta-STJs have achieved an energy resolution between $\sim 5$ and $\sim 15 \mathrm{eV}$ at $0.5 \mathrm{keV}$ and count rate capabilities of $\sim 5,000$ counts/s per pixel $[6,7]$. This is sufficient for $\mathrm{XAS}$ at synchrotron beam lines. Further increases in sensitivity now require primarily increased solid angle coverage.

Here we describe the development of a 112-pixel array of $208 \mu \mathrm{m} \times$ $208 \mu \mathrm{m}$ STJs that will replace the 36-pixel STJ detectors currently in use. It is based on a $\mathrm{Nb}(4 \mathrm{~nm})-\mathrm{Ta}(165 \mathrm{~nm})-\mathrm{Al}(50 \mathrm{~nm})-\mathrm{AlOx}-\mathrm{Al}(50 \mathrm{~nm})-\mathrm{Ta}(265 \mathrm{~nm})$ pentalayer process, and each row of 8 pixels shares the same ground, so that the entire array can be read out with 126 wires to the cryostat cold stage (Fig. 1, left). In addition, XIA LLC has built a compact 32-channel lownoise preamplifier that is computer-controlled and can be scaled to accommodate medium-size arrays (Fig. 1, right). Here we describe performance of the STJ detector array and the preamplifier in preparation for installation at the synchrotron.

\section{MULTICHANNEL PREAMPLIFIER}

The low-noise readout of an STJ X-ray detector with a typical dynamic resistance $>1 \mathrm{k} \Omega$ requires an FET with an input voltage noise $e_{n}$ $<1 \mathrm{nV} / \sqrt{\mathrm{Hz}}$, and a dc voltage bias to allow biasing in the low-noise regions between Fiske mode resonances. The voltage bias is implemented using a current-sensitive preamplifier with a feedback resistance $\mathrm{R}_{\mathrm{F}}=1 \mathrm{M} \Omega$ and a high open-loop gain $\mathrm{A}_{\mathrm{OL}}$, so that the dc load line impedance $\mathrm{R}_{\mathrm{F}} / \mathrm{A}_{\mathrm{OL}}$ is of order $\sim 1 \Omega$ (Fig. 2, center). A BF862 n-channel JFETs provides an input voltage noise $\mathrm{e}_{\mathrm{n}} \approx 0.8 \mathrm{nV} / \sqrt{\mathrm{Hz}}$, and is combined with a OP1 op-amp for a dc open-loop gain $\mathrm{A}_{\mathrm{OL}}>10^{6}$ [8]. A secondary feedback loop adjusts the JFET bias current so that its gate provides the desired STJ bias voltage without using a blocking capacitor [8]. The control point of this feedback loop, i.e.
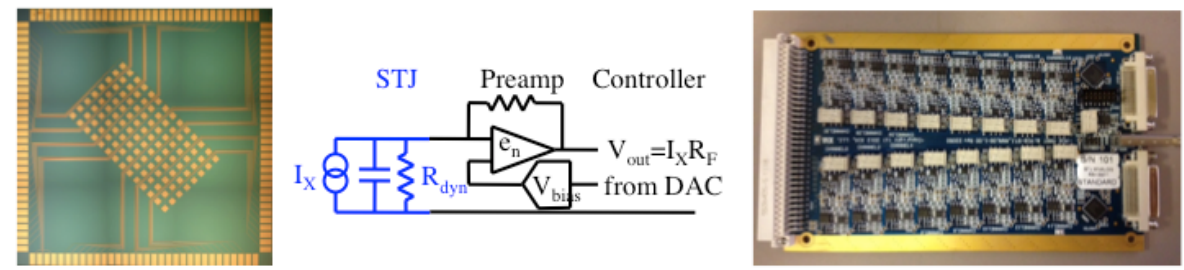

Fig. 1 (Left): Picture of 112-pixel STJ array. (Center): Schematic circuit diagram. (Right): Picture of preamplifier, with 16 channels on each side of the board. 


\section{2-pixel arrays of high-efficiency STJ X-ray detectors}

the STJ bias point, is set by a computer-controlled 16-bit digital-to-analog converter (DAC), contrary to our earlier pre-amplifiers where the bias had to be adjusted manually. The $\mathrm{I}(\mathrm{V}) \mathrm{s}$ of all 32 detector pixels can now be scanned simultaneously by scanning the DAC over the voltage range of interest and recording 1000 measurements of the output current for each channel; the average measures the sub-gap leakage current, their rms fluctuations the characteristic noise at each bias point (Fig. 2). The scan takes a few seconds, and several preamplifier boards can be operated in parallel in parallel so that the $\mathrm{I}(\mathrm{V}) \mathrm{s}$ of all the pixels in the array can be captured simultaneously. This simplifies the setup of the detector array.

The 112-pixel detector arrays were tested at $\sim 0.1 \mathrm{~K}$ in a two-stage adiabatic demagnetization refrigerator (ADR) at LLNL. The ADR temperature does not have to be regulated, as long as it is kept below $\sim 0.3 \mathrm{~K}$ such that the thermal quasiparticle background is negligible. Due to the limited number of wires in this test cryostat, only 32 pixels can be tested in each cool-down. Figure 2 (left) shows the I(V) characteristics of 26 pixels with high dynamic resistance at the optimum bias voltage of $\sim 100 \mu \mathrm{V}$. The other six were either open circuits or shorts. This suggests that the pentalayer process is intrinsically quite uniform, although processing can introduce pinhole defects in the junction barrier or open connections in the wiring, most likely at the $\mathrm{Nb}$ plugs between pixels that prevent charge sharing. The leakage current fluctuates slightly between different ADR cycles, indicating that different amounts of magnetic flux can be trapped when the Ta-STJs go though the superconducting transition during an ADR cycle.
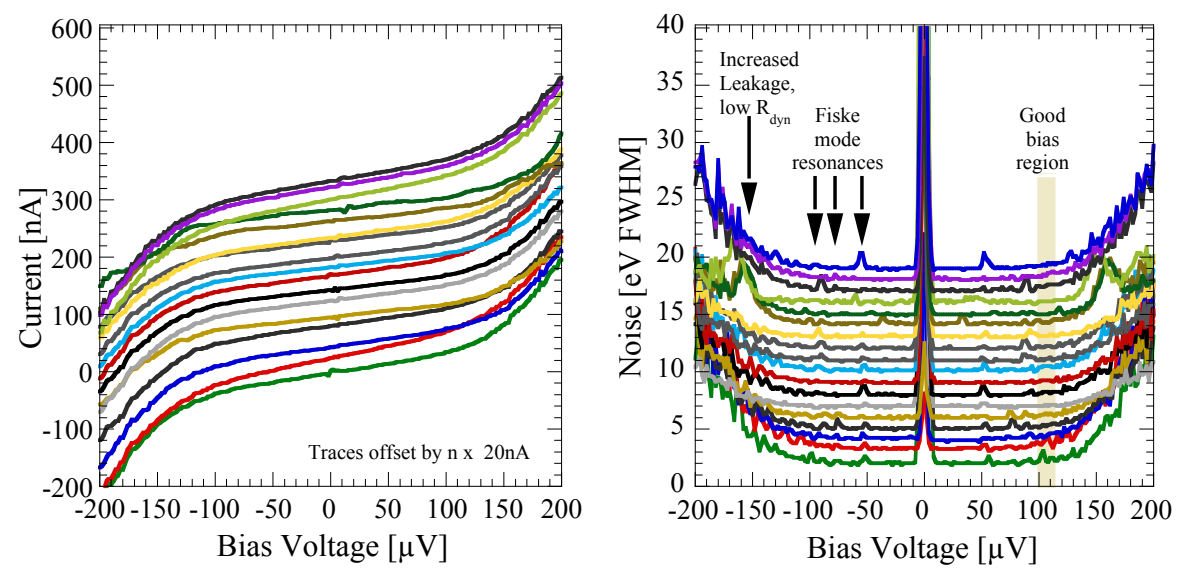

Fig. 2. Current-voltage (left) and noise characteristics (right) of 26 pixels in the array, taken simultaneously with the 32-channel preamplifier. Traces are offset vertically for clarity. 


\section{S. Friedrich et al.}

The STJ noise (Fig. 2, right), which has been scaled to $2.2 \mathrm{eV}$ in the low-noise region by comparison with the measured pulser width, is mostly determined by the dynamic resistance $\mathrm{R}_{\mathrm{dyn}}$ of the subgap current. It increases at the Fiske mode resonances and at bias voltages above $\sim 120 \mu \mathrm{V}$ where a reduced $\mathrm{R}_{\mathrm{dyn}}$ increases the noise contribution $\mathrm{e}_{\mathrm{n}} / \mathrm{R}_{\mathrm{dyn}}$ of the JFET's voltage noise $\mathrm{e}_{\mathrm{n}}$. Since the detector responsivity peaks around $\sim 100 \mu \mathrm{V}$, that region is preferred for high-resolution measurements. This bias point can be set remotely though the graphical user interface of the pre-amplifier.

\section{X-RAY RESPONSE}

The low-energy X-ray response of the arrays was tested at a two-stage $\mathrm{X}$-ray tube with a W/Ta primary target and various secondary targets on a target wheel. Figure 3 (left) shows the X-ray response of 26 pixels in the 112-pixel array to a $\mathrm{BN}+\mathrm{NiF}+\mathrm{SnZe}$ target on an $\mathrm{Al}$ target holder, taken simultaneously with the 32-channel preamplifier board. The responsivity is quite uniform, although it varies by $\pm 10 \%$ across the array, so that each pixel has to be calibrated individually. The inset shows the distribution of energy resolution among the 26 pixels, which varies between $\sim 8$ and $10 \mathrm{eV}$ FWHM at the $525 \mathrm{eV}$ oxygen $\mathrm{K}$ line. The fact that the energy resolution at the oxygen line is always better than at the lower-energy carbon and nitrogen lines indicates that the source is not truly monochromatic and that its line width contributes to the observed broadening.

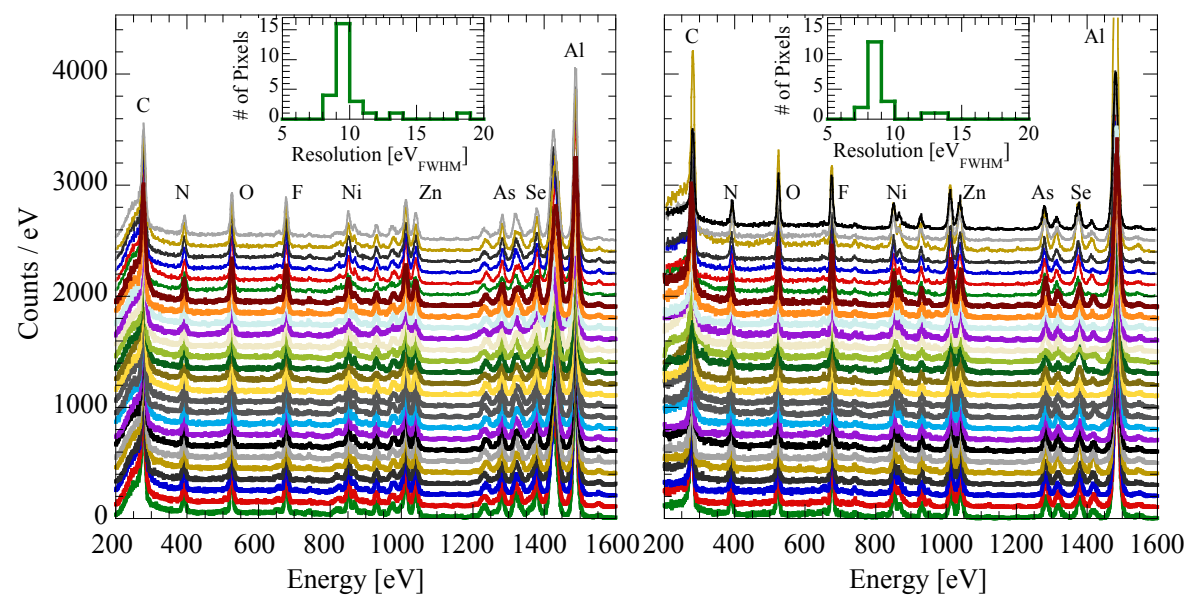

Fig. 3. X-ray response of 26 pixels to a $\mathrm{BN}+\mathrm{ZnSe}+\mathrm{NiF}$ sample on an $\mathrm{Al}$ sample holder, offset vertically for clarity. The spectra on the left were taken simultaneously using the new 32-channel XIA preamplifier, the ones on the right were taken sequentially with the older single-channel LLNL preamplifier. The inset shows a distribution of the pixel resolution at the $525 \mathrm{eV}$ oxygen $\mathrm{K}$ line. 


\section{2-pixel arrays of high-efficiency STJ X-ray detectors}

The $525 \mathrm{eV}$ oxygen line also has the smallest width in the control experiment where the X-ray response of the same 26 pixels was measured sequentially with the older single-channel preamplifier (Fig. 3, right). For these measurements, the entire signal waveform was captured, written to disc and subsequently processed off-line. This allowed using rise time discrimination to distinguish between absorption events in the top and the bottom electrode, and thus remove the line splitting artifact due to bottom layer absorption at higher energies. Although the average detector resolution is $\sim 1 \mathrm{eV}$ better in this particular run, this is most likely due to a lower leakage current during that ADR cycle, rather than to differences in preamplifier performance, since both preamplifiers have measured the same high energy resolution in other runs with identical junction $\mathrm{I}(\mathrm{V}) \mathrm{s}$.

Figure 4 shows the combined spectrum of the 26 pixels using the single-channel LLNL and the 32-channel XIA preamplifiers. Both spectra have an energy resolution of $9.5 \mathrm{eV} \mathrm{FWHM}$ at $\mathrm{O} \mathrm{K}$, and the background level is set by elastic scattering of the bremsstrahlung from the primary $\mathrm{X}$ ray target. One difference is that line splitting artifacts from base layer events (arrows) have been mostly suppressed during pulse-processing of the single-channel data. Another difference is the lower background below $\sim 250 \mathrm{eV}$, which is mostly due to substrate events whose slow rise time is less likely to produce a trigger signal in the XIA electronics.

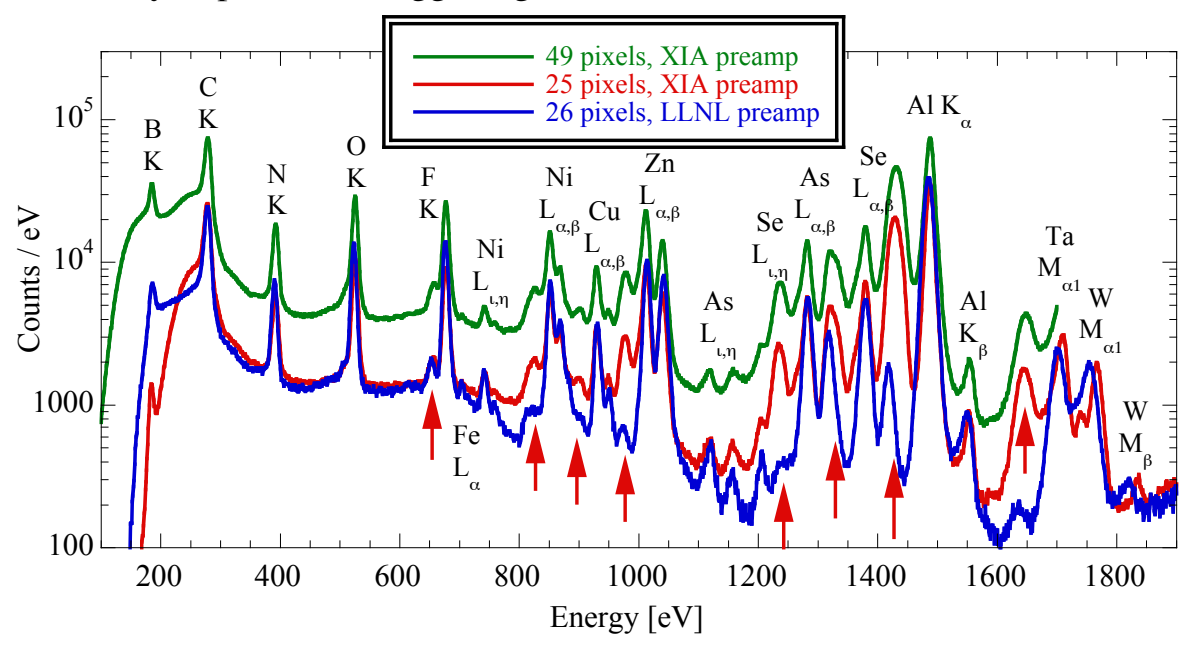

Fig. 4. Combined X-ray response of the STJ pixels in Fig. 3, measured once with the single-channel LLNL preamplifier (blue) and once with the 32-channel XIA preamplifier (red). For the LLNL preamplifier spectrum, rise-time discrimination has been used to suppress the line splitting artifact (arrows). The green spectrum shows the combined response of 49 STJ pixels from two separate cooldowns. 


\section{S. Friedrich et al.}

In the future we will integrate the 112-pixel array with four of the 32-channel preamplifiers in our liquid-cryogen-free ADR for synchrotron science. In addition, we are working on increasing the top Ta absorber film thickness and on rise time discrimination in the XIA pulse processing algorithm to remove the line splitting artifact for X-ray energies up to $>2 \mathrm{keV}$. This upgrade will increase the sensitivity of the instrument by almost an order of magnitude, and will enable the chemical analysis of dilute samples by PFY-XAS down to concentrations of $\sim 10$ to $100 \mathrm{ppm}$ [2].

\section{ACKNOWLEDGEMENTS}

This work was funded by the U.S. Department of Energy grants DESC0004359, DE-SC0006214, and DE-SC0002256. This work was performed under the auspices of the U.S. Department of Energy by Lawrence Livermore National Laboratory under Contract DE-AC52$07 N A 27344$.

\section{REFERENCES}

1. S. Friedrich, "Cryogenic X-ray detectors for synchrotron science", $J$. Synchrotron. Sci. 13, 159 (2006)

2. O. B. Drury, S. Friedrich, "Sensitivity and S/N-ratio of superconducting high-resolution X-ray spectrometers", IEEE Trans. Appl. Supercond. 15, $631(2005)$

3. V. Lordi, V. Gambin, S. Friedrich, T. Funk, T. Takizawa, K. Uno, J. S. Harris, "Nearest-neighbor configuration in (GaIn)(NAs) probed by X-ray absorption spectroscopy", Phys. Rev. Lett. 90, 145505 (2003)

4. K. Ndung'u, S. Friedrich, A. R. Gonzalez, A. R. Flegal, "Chromium oxidation by manganese (hydr)oxides in a California aquifer", Appl. Geochem. 25, 377-381 (2010)

5. M. Ohkubo, S. Shiki, M. Ukibe, N. Matsubayashi, Y. Kitajima, S. Nagamachi, "X-ray absorption near edge spectroscopy with a superconducting detector for nitrogen dopants in SiC", Sci. Reports 2, 831 (2012)

6. S. Friedrich, M. H. Carpenter, O. B. Drury, W. K. Warburton, J. Harris, J. Hall, R. Cantor, "New developments in STJ X-ray spectrometers for synchrotron science", J. Low Temp. Phys. 167, 741-747 (2012)

7. M. H. Carpenter, S. Friedrich, J. A. Hall, J. Harris, W. K. Warburton, R. Cantor, "Development of Ta-based STJ X-ray detector arrays", IEEE Trans. Appl. Supercond. 23, 2400504 (2013)

8. W. K. Warburton, J. Harris, M.H. Carpenter, L. Fabris, S. Friedrich, "Preamplifier development for superconducting tunnel junction array Xray detector electronics", in IEEE Nuclear Science Symposium (2011) 